\title{
The Referential Process as a Common Factor Across Treatment Modalities
}

\author{
Wilma Bucci ${ }^{1 凶}$
}

\begin{abstract}
The paper emphasizes the need for a process component in psychotherapy research, and the need for a theoretical framework within which process measures may be designed and validated. The referential process, defined in the context of multiple code theory, provides a general psychological framework for understanding the mechanisms of therapeutic change in different treatment modalities. The referential process includes three major phases: arousal/activation; narrative/symbolizing and reorganizing/reflection. The paper reviews the theoretical roots of psychodynamic treatments, and several forms of cognitive behavioral treatments, including schema therapy for borderline personality disorder and exposure treatments for post-traumatic stress disorder, and shows how the phases of the referential process provide common mechanisms of change in each of these approaches. Computerized measures of the referential process, in English and Italian versions, which have been applied and validated in clinical and experimental studies, are discussed.
\end{abstract}

Keywords: change mechanisms, referential process, computerized measures

The field of psychotherapy research is in need of scientific evidence concerning the basic processes underlying emotional disorders and therapeutic change. Such evidence is needed to guide clinical work, and to develop new and more effective treatments. Comparing the outcomes of competing theories is not useful if we do not identify the psychological mechanisms that bring about the observed results. To provide evidence concerning the psychological processes underlying therapeutic change and to develop new and more effective treatment forms, research is needed in both experimental and naturalistic contexts-in fields such as cognitive psychology, affective neuroscience, psycholinguistics, and developmental psychology; as well as in the therapeutic context.

In order to carry out such research in a way that will be applicable to clinical issues, an overarching theoretical framework is required. The study of internal psychological processes and variables, such as needs, desires, memories and perceptions, that figure in the psychotherapy process can be likened to the study of the fundamental constituents of matter in modern particle physics. Subatomic particles, like psychological processes, are known and assessed through the sig-

\footnotetext{
${ }^{1}$ Adelphi University, Derner Institute, Garden City (NY).

$\triangle$ Correspondence concerning this article should be addressed to Wilma Bucci, Adelphi University, Derner Institute, Garden City (NY), US, 11530.E-mail: wbucci@optonline.net
}

nals they send out and their observable effects. The advances in fields such as particle physics have occurred through development of a systematic theoretical framework that is constantly being re-examined and revised, with well defined concepts and relationships among the concepts; and with reliable and valid measures of external events from which inferences to these concepts and relationships can be made. The nature of the human emotional information processing system is at least as complicated as the nature of matter, but we know much less about it. Perhaps this is because it is more difficult to recognize how little we know in a scientific sense about emotional processes that we can feel operating within us; perhaps because it is so difficult to identify the level of explanation that is required.

Once we recognize how little we as psychotherapy researchers know about how psychotherapy works, we have taken one small step forward. The next step is to try to develop a coherent psychological theory, within which the concepts and processes that are studied can be systematically defined, in terms of one another and in terms of observable and measurable events; and to develop measures to assess the propositions and predictions of the theory.

\section{Concepts of multiple code theory and the referential process}

In previous work, I have proposed a general theory of emotional disorders and therapeutic change that is 
rooted in current psychological research and that provides a context for empirical research concerning the processes of therapeutic work, and their effects (Bucci, 1997, 2002). The clinical applications of this theory to date have been largely in the context of psychoanalytic theory and therapy. In this paper, I would like to expand the application of this model to identify basic psychological processes that are shared in several other treatment forms.

According to multiple code theory, emotional disorders result primarily from dissociation within emotion schemas, between the subsymbolic bodily, motoric, and sensory processes that constitute the affective core of the schema, and the objects and events of the world, particularly the interpersonal world, to which the subsymbolic processes have been linked (Bucci, 2007a, 2007b). When certain situations arise that trigger the affective core of a dissociated schema, the person is likely to experience painful affect whose meaning is not understood, and cannot be regulated. Dissociations may occur among emotion schemas as well as within them, involving failure of integration of different self-states and disconnections in the representation of the self.

People suffer not only because of the fundamental dissociations themselves, but because the solutions they have devised or the scenarios they have constructed to manage the painful affect have broken down, or are limiting or destructive in themselves. These strategies of self-regulation may take forms that are dangerous to oneself, such as addictions, somatization, eating disorders, and self cutting; as well as forms that are dangerous to others, including violent and abusive behavior and institutionalized expressions such as religious and political fanaticism.

The goal of treatment is reorganization of emotion schemas that have been dissociated and distorted. This requires taking in new information in the present about types of events and people that were threatening in the past, but that are no longer threats in the context of the person's current situation and current powers. The difficulty of treatment arises because dissociated schemas have been set up precisely to avoid situations associated with the dreaded schemas, so that there is no opportunity for the maladaptive expectations and beliefs to be disconfirmed.

In order for change to come about, some trace of the avoided schema must be activated in the session and in the relationship; but activated in such a way that the painful affect is reduced, and the situation experienced as new, rather than as reinforcement of previous maladaptive expectations. By this means, the tangle of avoidance and protection can be penetrated to some extent, and the schema can potentially be reconstructed rather than the dissociation being reinforced.

The communication that occurs between patient and therapist must function both to activate the problematic emotion schemas and to enter new information into them, and this must include providing a setting in the session that is experienced as different from the initial situation of pain or threat. The language of therapy must be of a nature that can connect to emotional experience-in some cases to activate, in some cases to regulate such experience.

\section{Phases of the referential process}

The mechanisms of therapeutic communication leading to change are defined within multiple code theory as the phases of the referential process, which has three major components, characterized as arousal/activation, narrative/symbolizing and reorganization/reflection:

(1) In the arousal/activation phase, traces of the problematic dissociated emotion schema are activated. The affective core is communicated primarily on bodily and motoric levels; this is what I have termed emotional communication (Bucci, 2001). The communication in subsymbolic form is shared by both participants. There is likely to be a fairly continuous flow of language during this phase, but the language that the patient speaksat least the semantic level of the language-is largely dissociated from the affective core that has been aroused.

(2) In the narrative/symbolizing phase, the patient talks about an episode of life, or tells a dream or fantasy whose connection to the problematic schema may not be recognized, or engages in and describes an event in the treatment relationship. The potential value of this process is to bring a representation of the problematic emotion schema into explicit and shareable symbolic form; this is likely to evoke corresponding experience in the therapist, enabling the therapist to resonate to the patient's experience.

(3) There is then an opportunity for the reorganizing/reflection phase in which a connection to the problematic schema may be identified and a new connection, a new meaning for the activation may be found.

The three components make up a schematic model; we expect that more effective sessions are more likely to be characterized by these three phases. The pattern may also occur sequentially across treatments, with several sessions manifesting the dominance of each phase. We do not expect the components of the process to occur in clear and orderly progression-there will be interruptions, avoidances and detours-but the patterning should emerge more clearly in more effective sessions and treatments.

\section{Back to the basics: Theoretical roots of contrasting therapeutic approaches}

Here I want to write briefly about the relation of the theoretical framework of multiple coding and the referential process to the theories of treatment underly- 
ing several of the major forms of psychotherapy being practiced today, including psychodynamic (PDT), cognitive behavioral (CBT) and exposure treatment forms (ET). When we examine the theoretical grounding of these approaches, we can see that each of them has its roots in basic theories of psychological functioning that were initially developed from fifty to a hundred or more years ago. While the techniques of treatment have evolved, the theoretical roots have generally not been subjected to critical examination and revision.

\section{Theoretical roots of psychoanalytic and psychodynamic treatments}

The roots of PDT lie in psychoanalytic principles, based initially on a hybrid psychological/biological model of more than a century ago, the energy theory of Freud's metapsychology. This theoretical level of explanation has been explicitly rejected or at least ignored by psychoanalytic clinicians today, although many concepts associated with the energy theory, such as ego, id, repression, the primary and secondary processes and the concept of psychodynamic itself, are retained by clinicians without acknowledgment of their source. I have previously discussed these concepts and the problems associated with them, in the context of current research in cognitive science and related fields (Bucci, 1997).

Several core principles of the psychoanalytic theory of pathology and treatment can be identified as this has evolved in clinical work over the past century. These fundamental principles are shared by most psychodynamic clinicians, with variants of each. According to most psychoanalytic and psychodynamic approaches, emotional disorders are caused by ideas or feelings that are split off from, not integrated with the network of associations that regulate and organize life. The splitting off may be characterized as repression (unconscious fantasies, wishes, desires) or dissociation (involving different modes or states of functioning) and may be caused by acute or chronic developmental trauma, hereditary weakness or some combination of all these. The ideas or feelings that are unintegrated and kept out of awareness influence behavior and cause physical and psychological symptoms. Cure [change] occurs through talking in the context of the therapeutic relationship so that the idea is brought to awareness, connected to the contents of conscious thought, seen in the light of reality, able to be regulated through being connected to the network of associations that organize life.

Ernst Kris, who is associated with the ego psychology version of the classical psychoanalytic approach has provided an outline of psychoanalytic process in his famous characterization of the "good hour:"

Many a time the "good hour" does not start propitiously. It may come gradually into its own, say after the first ten or fifteen minutes, when some recent experience has been recounted, which may or may not refer to yester- day's session. Then a dream may come, and associations, and all begins to make sense. In particularly fortunate instances a memory from the near or distant past, or, suddenly, one from the dark days may present itself with varying degrees of affective charge. At times new elements are introduced as if they had always been familiar, so well do they seem to fit into the scheme of things. And when the analyst interprets, sometimes all he needs to say can be put into a question. The patient may well do the summing up by himself, and himself arrive at conclusions (Kris, 1956, p. 446).

Almost half a century later, Philip Bromberg has described analytic work from the perspective of the current relational approach with quite different emphasis but in terms of a related sequence of processes:

In the proper analytic setting, there is a chance, with the analyst, for the dissociated domains of self to play out aspects of unsymbolized experience that will allow motoric, affective, imagistic, and verbal elements to coalesce with relevant narrative memory in the context of something formerly unthinkable: A perceptual experience of the patient-analyst relationship as a dyadically constructed illusion, linking internal truth with a new, self-consistent, more flexible version of external reality (Bromberg, 1998, p. 183).

While these psychoanalytic approaches differ in many ways, they share a recognition of several basic phases of analytic work:

(a) A phase of unsymbolized experience, characterized by Kris as ten or fifteen minutes of nothing much happening; by Bromberg as active playing out of unsymbolized experience.

(b) Emergence of content that can be symbolized and verbalized. Kris speaks of telling recent experiences, dreams and memories; Bromberg talks about the bodily elements coalescing with relevant narrative memory.

(c) Symbolized contents are available to selfreflection. Kris speaks of interpretation, summing up and conclusions; Bromberg talks of linking internal truths with new versions of external reality.

\section{Theoretical roots of cognitive behavioral treatments}

The basic premises of cognitive therapy were developed by a psychoanalyst, in relation to and in contrast toperhaps in reaction to-the premises underlying psychoanalysis. From the perspective of the 1960s, Beck's theory of treatment was seen as revolutionary in three major ways: (1) emphasizing the patient's life outside sessions rather than focusing on the therapeutic relationship (or transference), (2) focusing on the present rather than early childhood, and (3) focusing on the contents of consciousness rather than the unconscious.

The model of psychopathology in the cognitive behavioral approach is based on the premise that emotional distress and problems in adaptation are caused by false beliefs and faulty information processing 
strategies. The central psychological construct is the schema; these are conceptualized as "stored bodies of knowledge" that "are hypothesized to develop from interactions with the environment, primarily those interactions that occur during childhood," and that "interact with incoming information to influence selective attention and memory search" (Scher, Segal, \& Ingram, 2006, p. 29). According to Beck's theory of cognitive specificity (Beck, 1976) each emotion is paired with particular cognitive themes built on these schemas-depression with cognitive themes of pessimism, self-criticism and hopelessness; anxiety with themes of threat, danger and vulnerability, overestimation of danger, underestimation of powers and resources; anger with themes of violation and hurt, and perception of others as malevolent. Symptoms are viewed as dependent on the beliefs and strategies incorporated in the schemas.

The treatment focuses on correcting the false beliefs, and bringing about change in the individual's strategies of responding to the events of life by this means. The therapist encourages clients to use their own behaviors to test their beliefs, for example to carry out "experiments" to test the accuracy of negative beliefs. The treatment also uses techniques such as empirical analysis and logical discourse.

The psychological basis for CBT lies in appraisal theory, stemming from the work of researchers such as Lazarus $(1968,1982)$. According to this approach, emotional experience begins with a cognitive evaluation of the significance of an event, followed by additional evaluations of the situation that may encompass the physiological activation, and leading to selection of a response. The processes underlying appraisal techniques have been questioned and revised in the fields of cognitive science and neuroscience since their initial formulation. Zajonc (1984) provided experimental evidence that affective response may precede and direct cognitive appraisal, rather than cognition directing affect. Recent work in affective neuroscience by Le Doux (2002), Damasio (1994, 1999), Ochsner and Gross (2008) and many others have provided far more complex perspectives on the interaction of emotion, behavior and cognition than was represented in the early appraisal theories. Some of the new findings are represented in the variants of cognitive behavioral treatments that have emerged more recently.

\section{Schema therapy for borderline personality disorder}

As Klosko and Young (2006) have pointed out, patients with characterological disorders, such as borderline personality disorder (BPD) tend to lack certain capabilities that are generally required for successful application of cognitive behavioral treatments. They are likely to have chronic, pervasive problems in their relationships and in their work. Their problems are not well formulated and they avoid addressing these problems. They cannot engage in a relationship of trust with the therapist, and logical discourse has little effect in bringing about recognition of their difficulties and in effecting change.

A form of treatment termed schema therapy has been developed for working with such patients (Young \& Lindemann, 1992). Schema therapy differs from standard forms of CBT in a number of major ways, particularly in its focus on developing the therapeutic relationship and on exploring the patient's traumatic childhood experiences. (With these two changes, schema therapy constitutes a counterrevolution in the CBT camp, undoing two of the basic principles distinguishing CBT from psychoanalysis, as introduced by Beck).

The concept of schema mode is central to the formulation of BPD as understood in this approach. The schema mode is defined by Klosko and Young (2006, p. 276) as "those schemas or coping responsesadaptive or maladaptive-that are currently active for an individual" contrasting with the general concept of schemas, which have trait-like status. Patients with BPD are characterized by relatively rapid shifting among modes, often with extreme affective components. A number of specific modes have been identified; these include: abandoned child, angry and impulsive child, punitive parent, detached protector, and healthy adult. These draw from psychoanalytic object relations theorists such as Winnicott (1971), as well as the attachment theory of Bowlby (1969) and other developmental theorists.

In the beginning of treatment, in a phase that is termed bonding and emotional regulation, the patient is primarily in what schema therapists characterize as the "abandoned child" mode. As Klosko and Young describe the work in this phase:

The therapist begins to reparent the patient's abandoned child, providing safety and emotional holding... The goal is for the therapist to create an environment that is a partial antidote to the one the patient knew as a child-one that is safe, nurturing, protective, forgiving and encouraging of self-expression (Klosko \& Young, 2006, p. 280).

Somewhat later in treatment, when the therapeutic bond is more secure, and the patient is strong enough not to decompensate, the therapist attempts in a variety of different ways to enable the patient to access and work through images of upsetting events from childhood. This involves a phase that is termed the experiential work. As Klosko and Young say: "The therapist enters the images and does whatever a good parent would have done: removes the child from the scene, confronts the perpetrator, stands between the perpetrator and the child, or empowers the child to handle the situation" (Klosko \& Young, 2006, p. 287).

As patients develop trust in the stability of the relationship, they become more able to utilize standard cognitive-behavioral techniques, in the stages of cognitive work and behavioral work; these include education, reappraisal, and practicing self-regulation techniques. If the therapist focuses on cognitive themes or 
introduces cognitive restructuring techniques too early they are unlikely to be effective; the patient is likely to reject them as cold and mechanical, and to reject the treatment.

\section{Exposure therapies for post-traumatic stress disorder}

Exposure therapies, particularly involving prolonged exposure (PE), have been identified as having strong research support for treatment of post-traumatic stress disorder (PTSD) (Baker, McFall, \& Shoham, 2008; Foa et al., 2005). Some therapies for treatment of other anxiety disorders including phobias, panic disorder, obsessive-compulsive disorder (OCD), and generalized anxiety disorder (GAD) also incorporate components of exposure to feared situations. Exposure therapy has its roots in conditioning theory, particularly Mowrer's (1960) two factor conditioning theory, involving CS-UCS (S-S) as well as traditional stimulus response (S-R) connections, and in the information processing theory of Lang (1977) as outlined by Foa and Kozak (1986).

In PTSD, anything associated with the trauma may activate the fear network; the fear network is thought to be broad, stable, easily accessed, but is difficult to change because of the escape and avoidance behavior that is an intrinsic component. The network is built not only by the initial traumatic event and conditioned stimuli associated with it, but also through a wide range of information, including reports of events by others or emergence of new data in life experience concerning the implications of events.

Modifying a fear structure requires what Foa and Kozak refer to as emotional processing:

\begin{abstract}
We propose that regardless of the type of therapeutic intervention selected, two conditions are required for the reduction of fear. First, fear-relevant information must be made available in a manner that will activate the fear memory. Indeed, as suggested by Lang (1977), if the fear structure remains in storage but unaccessed, it will not be available for modification. Next, information made available must include elements that are incompatible with some of those that exist in the fear structure, so that a new memory can be formed. This new information, which is at once cognitive and affective, has to be integrated into the evoked information structure for an emotional change to occur (Foa \& Kozak, 1986, p. 22).
\end{abstract}

The techniques of treatment are based on gradual confrontation of the traumatic memory, through imaginal or in vivo exposure. Imaginal exposure to the trauma memory involves either telling the memory in the session or writing an account at home that is then brought in and read in the session. The patient is asked to tell or write exactly what happened with as many details as possible, including sensory details as well as thoughts and feelings about the event. In telling the memory in the session, $s$ /he may be asked to speak in the present tense. In writing the memory, $s /$ he is asked to hand write rather than type the report, to enable more subjective connection, and to do the writing when $s / h e$ is at home and alone so that $s / h e$ can cry and feel other emotions without being interrupted or embarrassed (Resick et al., 2007).

The arousal of a fear or other emotion structure in a context different from the one in which the painful experience was initially constructed constitutes the active psychological ingredient of the exposure treatments. In practice, many patients in exposure treatments do not feel sufficiently stable to manage the reexperiencing of somatic symptoms that occurs, including autonomic disregulation, intrusive sensory experiences and involuntary movements, any of which can involve extreme distress, and lead to symptom exacerbation. To protect against the anticipated decompensation, patients are likely to withdraw emotionally or terminate prematurely. Significant level of treatment drop-out and high nonresponse rates have been found in studies of exposure treatments (Schottenbauer et al., 2008). As Cook et al. (2004) point out, care needs to be taken in applying PE so that "the rigors of exposure are tempered by empathy and a collaborative therapeutic alliance" (p. 381).

\section{The referential process in alternate treatment forms}

As the brief outline above has indicated, the several treatment forms that have been discussed have their roots in theoretical foundations that are no longer supported as such in current scientific work. These include the energy theory of the psychoanalytic metapsychology, the appraisal theory underlying CBT, and Mowrer's (1960) conditioning theory. The various therapeutic approaches have continued to develop and advance considerably in recent years, mostly in the clinical domain and largely without change in basic theory or evidence from research. The psychoanalytic approach has begun to incorporate an increased emphasis on the importance of the therapeutic relationship, and the role of nonverbal communication in the treatment process, with support from attachment research and related fields. Exposure treatments increasingly incorporate changes in meaning of the feared events-in conditioning terms building new S-S connections; in terms of Lang's (1979) bioinformational model entering new information into memory networks. Both systems of S-S connections and memory networks are conceptually related to the general construct of the schema that is included in other forms of the cognitive-behavioral approach as well as in psychodynamic treatments, and that provides a potentially useful psychological common ground, with research support in several experimental fields.

In this paper I will focus on the application of the referential process in the several treatment forms that have been discussed here. The three components of the process are represented in different ways and to varying degrees in each of these treatments.

Psychoanalytic/psychodynamic treatments. The therapeutic application of the referential process was 
initially examined in relation to psychodynamic treatment forms and can be seen most fully there. As illustrated earlier in the contrasting writings of Kris and Bromberg (1998), the several variants of psychoanalytic and psychodynamic treatment generally accept some version of the following model of the treatment process: (1) an opening phase of activation of the affective core of an emotion schema in which the patient appears to be struggling with his ideas or feelings, not acknowledged or explicitly addressed, (2) a phase of narrative and symbolizing that includes verbal reports of dreams, fantasies, and memories, with affective charge as well as enactment of activated schemas in the relationship, and (3) a phase of reflection on the contents of the associations, leading to reorganization of the psychic structures that have been dissociated. The classical theory emphasizes interpretation and insight in the reflection phase; the relational approach emphasizes patient and analyst accessing and experiencing unsymbolized aspects of the process that is going on between them, rather than conceptualizing the process in terms of the patient's internal struggles. The three phases apply in short as well as long-term psychodynamic treatment forms.

General CBT approach. In the standard CBT paradigm the phase of activation may occur in some form in the "experiments" that the clients are encouraged to carry out to test their beliefs. The narrative symbolizing component may occur in the session as the client talks about these events. The effectiveness of treatment has been shown to be related to the degree to which the client is able to talk about experience in a vivid and connected rather than ruminative and abstract way (Borkovec, Roemer, \& Kinyon, 1995). The reorganizing phase is dominant in the CBT treatments, including discussion of cognitive themes, examination of evidence from "experiments," and questioning of expectations and beliefs.

Schema therapies for BPD. The activation component is dominant in the bonding and emotional regulation phase of schema therapies. The therapist is seeking to communicate in nonverbal and verbal ways, to create a context that the patient can experience as different from the initial setting in which the maladaptive emotion schema was developed. In the phase of experiential work, the processes of narrative symbolizing occur, as the therapist enables the patient to access and talk about images of upsetting events from childhood. The therapist may also enter the images to reparent the child and relive the events in the new context of the relationship. A reorganizing phase can then occur in the phases of cognitive and behavioral work that include use of cognitive techniques such as education and reappraisal.

Exposure therapies for PTSD. The activation and narrative symbolizing components occur interactively through telling or writing the traumatic memory repeatedly in concrete specific sensory detail. The narrative may serve as a stimulus for activation of a painful schema as well as an expression of the schema that has been aroused. The effect of the narrative in evoking emotion constitutes the power but also the danger of the imaginal exposure treatment forms. Reorganizing occurs through questioning false beliefs, as in other CBT forms. In the context of the conditioning paradigm that underlies exposure treatment, change is also expected to occur as a form of extinction, by means of habituation through repetition.

\section{Identification and assessment of common processes}

Our basic claims are that effective sessions (and treatments) in different treatment forms are characterized by occurrence of the three phases, essentially sequentially, although with interactions and interruptions; and that we can differentiate more or less effective treatments based on these measures. There must be some representation of each of the three phases, in some form in a session that is effective: the painful emotion schema must be activated, at least in trace form; the schema must be expressed in a narrative that emerges in a vivid and evocative form, or in an interaction of the relationship; some evaluation and reflection on the narrative then needs to occur. Implicit in this claim is the necessary role of the therapist and the therapeutic relationship in enabling these phases and the progression through them.

\section{Computerized measures of the referential process}

Within the context of multiple code theory, computerized linguistic measures have been designed to represent the three phases of the referential process. These measures have been applied and validated in several clinical and experimental studies in English (Bucci \& Maskit, 2007; Bucci, Maskit, \& Hoffman, 2012). The English versions of the dictionaries are defined in our website www.thereferentialprocess.org. Italian versions of the measures have been applied to three short-term psychodynamic treatments (Mariani, Maskit, Bucci, \& De Coro, 2013).

Briefly, the Arousal phase is characterized by high Disfluency (DF; um, uh, like, well, kind of etc. etc.), high mixed affect without specific valence, somatization words, and by low Referential Activity (RA; narrative) and low Reflection (REF). For example:

Really I- I don't think I- I had that feeling before. I ah, I
mean as yet. I've ah, well, this really feels like I'm saying
something that ah, is a part of me, really, or ah, / / / / it
feels like I'm ah, sort of have ah, I don't know.
(The series of slashes represent pauses of several sec-
onds). ${ }^{1}$

The Narrative/Symbolizing mode is characterized

\footnotetext{
${ }^{1}$ All of the examples here are taken from verbatim therapy transcripts in our research archives, with somewhat varying transcription methods; all possible identifying information has been removed or edited.
} 
by vivid and concrete imagery and action, represented in high RA. Reflection is low; speech is fluent with low $\mathrm{DF}$ and little pausing, as in the following:

I remember one house I went into, it was, it was in the winter and it was really a little shack up on the roof of another house, and you took your life in your hands climbing up the steps. You had to go up outside steps on the roof, and there were rats and dogs and cats all around and when I got in, the woman I went to see was well, really sleeping off a hangover from a Sunday night party, and she was sleeping in all her clothes in a dirty, filthy bed in the kitchen, and a dirty baby was running around, probably hadn't been attended and it was a filthy place and I sat and talked to her alone. And she told me in a very calm voice something about shooting her sister-in-law.

The reorganizing phase is characterized by high Reflection, low RA, affect words with specific valence and some Disfluency:

So I suppose there again, it's your approving or disapproving. (Pause) And of course, what it boils down to is I'm sure you're going to (chuckle) disapprove, so I'm afraid of saying them. (Pause, stomach rumble) It's funny how we-I asked John about it too-and, and, I don't know, we, we've been debating how much we should talk about our experiences anyway, and I imagine that we really don't s- speak much about it.

We are also starting to look at pausing and speech rhythms in the audiotapes of sessions; these could be important as well in differentiating the phases.

\section{Conclusion: the need for a process component in psychotherapy research}

The field of psychotherapy research has recently focused on outcome rather than process studies, with outcome mainly evaluated in terms of symptoms and behaviors. This emphasis has occurred for many reasons, including professional, ethical (and financial) considerations-as well as the fact that process research is difficult, time consuming and expensive. Many well designed outcome studies do include a process component, but this has been largely in the service of confirming adherence to the therapeutic approaches whose outcomes are being compared.

A recent paper by Barber, Barrett, Gallop, Rynn, and Rickels (2011) is a model of a well designed study exemplifying the comparative outcome approach. The NIMH supported study, conducted at the University of Pennsylvania from November 2001 through June 2007 compared three treatment conditions: supportive-expressive psychodynamic therapy; pharmacotherapy plus clinical management; and placebo plus clinical management for major depressive disorder. All groups improved over the 16 weeks of the treatment, with strong effect sizes-and no between-group differences. These null results are similar to those found in the 1985 NIMH supported TDCRP (treatment of depression collaborative research program), which compared 16 week treatments in medication, CBT, IPT and placebo and found no significant differences among the three active treatments at termination and no differences among all four treatment conditions at follow-up (Elkin, 1994; Elkin et al., 1989).

I suggest that it is time to move on to a systematic process oriented approach to assessing treatment effects. Such studies can potentially be incorporated in outcome studies such as the TDCRP or Penn studies, and can provide information as to the members of each group who were or were not helped by the treatment, and how these effects occurred. Studies by Blatt and colleagues (Blatt, Zuroff, Hawley, \& Auerbach, 2009) have been successful in identifying pretreatment personality differences of patients (and therapists) that impacted the treatment effects. In a similar approach, common process factors can be identified that relate to therapeutic change in each treatment approach. The development of computerized linguistic measures has made this approach more feasible. In addition, the use of speech recognition software can under some circumstances be used to further improve the efficiency of process research based on transcriptions of sessions.

The fundamental issues of psychotherapy research that need to be understood in psychological terms include characterizing the problems that bring patients to treatment, identifying the processes that bring about change, and knowing when change has occurred. To address these issues we need to follow the strategies of other scientific fields: to develop a systematic theoretical framework that is constantly being reexamined and revised, in both experimental and clinical contexts; and to develop reliable and valid measures from which systematic inferences to the concepts of the theory may be made. These challenges may be at least as great as finding the particle that explains the nature of mass, but if physicists have hope in their quest, we can too.

\section{References}

Baker, T. B., McFall, R. M., \& Shoham, V. (2008). Current status and future prospects of clinical psychology: Toward a scientifically principled approach to mental and behavioral health care. Psychological Science in the Public Interest, 9(2), 67-103. doi: 10.1111/j.1539-6053.2009.01036.x

Barber, J. P. Barrett. M. S., Gallop, R., Rynn, M. A., \& Rickels, K. (2012). Short-term dynamic psychotherapy versus pharmacotherapy for major depressive disorder: A randomized, placebo-controlled trial. Journal of Clinical Psychiatry, 73(1), 66-73. doi:10.4088/JCP.11m06831

Beck, A. T. (1976). Cognitive Therapy and the Emotional Disorders. New York: International Universities Press.

Blatt, S. J., Zuroff, D. C., Hawley, L. L., \& Auerbach, J. S. (2009). Predictors of sustained therapeutic change. Psychotherapy Research, 20(1), 37-54. doi: 10.1080/10503300903121080

Borkovec, T. D., Roemer, L., \& Kinyon, J. (1995). Disclosure and worry: Opposite sides of the emotional processing coin. In J. W. Pennebaker (Ed.), Emotion, Disclosure and Health (pp. 47-70). Washington, DC: American Psychological Association Books. 
Bowlby, J. (1969). Attachment and loss. (Vol. 1. Attachment). New York: Basic Books.

Bromberg, P. M. (1998). Standing in the Spaces. Hillsdale, NJ: The Analytic Press.

Bucci, W. (1997). Psychoanalysis and Cognitive Science: A multiple code theory. NY: Guilford Press.

Bucci, W. (2001). Pathways of emotional communication. Psychoanalytic Inquiry, 20, 40-70. doi: 10.1080/07351692109348923

Bucci, W. (2002). The Referential Process, consciousness, and the sense of Self. Psychoanalytic Inquiry, 22, 766-793. doi: $10.1080 / 07351692209349017$

Bucci, W. (2007a). Dissociation from the perspective of multiple code theory: Part I: Psychological roots and implications for psychoanalytic treatment. Contemporary Psychoanalysis, 43(2), 165-184.

Bucci, W. (2007b). Dissociation from the perspective of multiple code theory: Part II: The spectrum of dissociative processes in the psychoanalytic relationship. Contemporary Psychoanalysis, 43(2), 305-326.

Bucci, W., \& Maskit, B. (2007). Beneath the surface of the therapeutic interaction: The psychoanalytic method in modern dress. Journal of the American Psychoanalytic Association, 55, 1355-1397. doi: 10.1177/000306510705500412

Bucci, W., Maskit, M., \& Hoffman, L. (2012). Objective measures of subjective experience: the use of therapist notes in process-outcome research. Psychodynamic Psychiatry, 40(2), 303-340. doi: 10.1521/pdps.2012.40.2.303

Cook, J. M., Schnurr, P. P., \& Foa, E. B. (2004). Bridging the gap between Post Traumatic Stress Disorder research and clinical practice: the example of exposure therapy. Psychotherapy: Theory, Research, Practice, Training, 41(4), 374-387. doi 10.1037/0033-3204.41.4.374

Damasio, A. R. (1994). Descartes' Error: Emotion, reason and the human brain. NY: Avon Books, Inc.

Damasio, A. R. (1999). The Feeling of What Happens. NY: Harcourt Brace \& Co.

Elkin, I. (1994) The NIMH Treatment of Depression Collaborative Research Program: Where we began and where we are. In A. E. Bergin \& S. L. Garfield (Eds.), Handbook of Psychotherapy and Behavior Change, 114-139. NY: Wiley \& Sons.

Elkin, I., Shea, M. T., Watkins, J. T., Imber, S. D., Stosky, S. M., ..., Parloff, M. B. (1989). National Institutes of Mental Health Treatment of Depression Collaborative Research Program: General effectiveness of treatments. Archives of General Psychiatry, 46(11),71-982. doi:10.1001/archpsyc.1989.01810110013002.

Foa, E. B., Hembree, E. A., Cahill, S. P., Rauch, S. A., Riggs, D. S., \& Feeny, N. C. (2005). Randomized trial of prolonged exposure for posttraumatic stress disorder with and without cognitive restructuring: Outcome at academic and community clinics. Journal of Consulting and Clinical Psychology, 73(5), 953-964. doi: 10.1037/0022-006X.73.5.953

Foa, E. B., \& Kozak, M. J. (1986). Emotional processing of fear: Exposure to corrective information. Psychological Bulletin, 99(1), 20-35. doi: 10.1037//0033-2909.99.1.20
Klosko, J., \& Young, J. (2006). Cognitive therapy of Borderline Personality Disorder. In R. L. Leahy (Ed.), Contemporary Cognitive Therapy: Theory, Research, and Practice (pp. 269298). NY: Guilford Press.

Kris, E. (1956). On some vicissitudes of insight in psychoanalysis. International Journal of Psychoanalysis, 37, 445-455.

Lang, P. J. (1977). Imagery in therapy: An information processing analysis of fear. Behavior Therapy, 8, 862-886. doi: 10.1016/S0005-7894(77)80157-3

Lang, P. J. (1979). A bio-informational theory of emotional imagery. Psychophysiology, 16(6), 495-512. doi: 10.1111/j.14698986.1979.tb01511.x

Lazarus, R. S. (1968). Emotions and adaptation: Conceptual and empirical relations. In W. J. Arnold (Ed.), Nebraska Symposium on Motivation. Lincoln: University of Nebraska Press.

Lazarus, R. S. (1982). Thoughts on the relations between emotion and cognition. American Psychologist, 37, 1019-1024. doi: 10.1037/0003-066X.37.9.1019

LeDoux, J. E. (2002). The synaptic self. New York: Viking.

Mariani, R., Maskit, B., Bucci, W., \& De Coro, A. (2013). Linguistic measures of the referential process in three shortterm psychodynamic treatments: The English and Italian versions. Psychotherapy Research, 23(4), 430-447. doi: 10.1080/10503307.2013.794399

Mowrer, O. H. (1960). Learning theory and behavior. New York: Wiley.

Ochsner, K. N., \& Gross, J. J. (2008). Cognitive emotion regulation: Insights from social cognitive and affective neuroscience. Current Directions in Psychological Science, 17, 153-158. doi:10.1111/j.1467-8721.2008.00566.x

Resick, P. A., Mondson, C. M., \& Chard, K. M. (2007). Cognitive Processing Therapy: Veteran/military version. Washington, DC: Department of Veterans' Affairs.

Scher, C. D., Segal, C. V., \& Ingram, R. E. (2006). Beck's theory of depression: Origins, empirical status, and future directions for cognitive vulnerability. In R. L. Leahy (Ed.), Contemporary Cognitive Therapy: Theory, Research, and Practice (pp. 27-44). NY: Guilford Press.

Schottenbauer, M. A., Glass, C. R., Arnkoff, D. B., Tendick, V., \& Gray, S. H. (2008). Nonresponse and dropout rates in outcome studies on PTSD: Review and methodological considerations. Psychiatry, 71(2), 134-168. doi: 10.1521/psyc.2008.71.2.134

Winnicott, D. W. (1971). Playing and Reality. New York: Basic Books.

Young, J., \& Lindemann, M. D. (1992). An integrative schemafocused model for personality disorders. Journal of Cognitive Psychotherapy: An International Quarterly, 6, 11-24.

Zajonc, R. B. (1984). The interaction of affect and cognition. In K. R. Scherer \& P. Ekman (Eds.), Approaches to emotion (pp. 239-246). Hillsdale, NJ: Lawrence Erlbaum Associates, Inc.

Received January 16, 2012

Revision received April 9, 2012

Accepted February 23, 2013 